\title{
INTEGRATED SURFACE-MICROMACHINED MASS FLOW CONTROLLER
}

\author{
Jun Xie, Jason Shih and Yu-Chong Tai \\ Caltech Micromachining Lab, Electrical Engineering 136-93, \\ California Institute of Technology, Pasadena, CA 91125, USA
}

\begin{abstract}
An integrated surface-micromachined mass flow controller (MFC) that consists of an electrostatically actuated microvalve and a thermal flow sensor is presented here. With a unique design and utilizing a multilayer Parylene process, the active microvalve and the flow sensor are integrated onto a single chip to perform closed-loop flow control. Sensitivity of the flow sensor is $55 \mu \mathrm{V} /(\mu \mathrm{L} / \mathrm{min})$ for airflow and $12.2 \mu \mathrm{V} /(\mathrm{nL} / \mathrm{min})$ for water. The valve is actuated with a $10 \mathrm{kHz} \mathrm{AC}$ signal and an applied pressure of $21 \mathrm{kPa}$ can be sealed with an actuation voltage of $200 \mathrm{~V}_{\text {peak }}( \pm 200 \mathrm{~V})$. For flow control, both Pulse Width Modulation (PWM) and actuation voltage adjustment are demonstrated. PWM shows better performance in terms of controllability and linearity.
\end{abstract}

\section{INTRODUCTION}

Total integration of many microfluidic devices (e.g., micro pump, valve and flow meter) onto a single chip has been a long sought goal in the microfluidics field [1]. While many devices have been demonstrated using a variety of technologies, incompatibility among different fabrication technologies can create problems during integration. It has been a long-term goal for us to develop a multilayer Parylene surface micromachining technology [2] that allows the total integration of various microfluidic devices.

Flow control is an important function that many microfluidic systems must have. Because of low power consumption and easy implementation, electrostatically actuated microvalves are attractive and have been widely used in flow control $[1,3,4]$. Unlike those previous microvalves, our new electrostatically actuated microvalve is entirely surface micromachined using Parylene technology. Moreover, with the versatility of the multilayer process, a thermal based flow senor is also integrated with the microvalve for flow measurement and closed-loop feedback flow control. Figure I shows the fabricated micro flow controller on a PCB-mounted microfluidic chip.

\section{DESIGN}

Figure 2 shows the design of the micro mass flow controller and its operation principle. The design takes advantage of the versatility and flexibility of the multilayer Parylene process. The microvalve is realized by using electrostatic force to actuate a circular Parylene

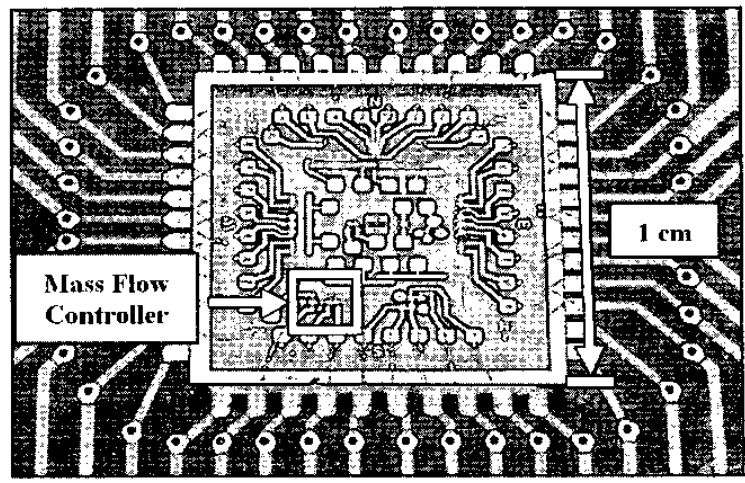

Figure 1. Integrated microfluidic chip mounted on PCB. Micro flow controller

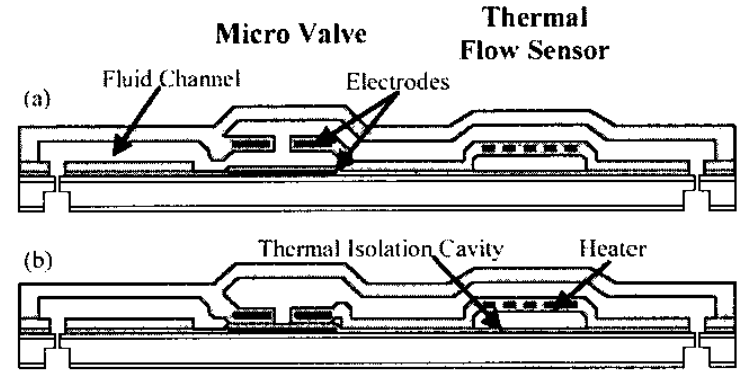

Figure 2. Operation principle and design of the micro flow controller. (a) Valve Open, (b) Valve Close.

membrane. An applied voltage moves the membrane in contact with the valve seat and closes the fluid pathway. So this microvalve is a normally open device. All the electrodes are encapsulated by Parylene to achieve electrical insulation. Experimentally, it is found that the breakdown field of Parylene is about $200 \mathrm{~V} / \mu \mathrm{m}$, which allows good electrical insulation with just a thin Parylene layer [5]. Here, the dimensions of the moving membrane (200 $\mu \mathrm{m}$ diameter, $2 \mu \mathrm{m}$ thick) are carefully chosen so the diaphragm won't be too flexible as to stick to the bottom during drying nor too stiff to bend during operation. The flow sensor is thermally based [6]. In CC (Constant Current) mode, the flow rate is related to the voltage drop across the resistive heater due to heat transfer between the heater and fluid inside the channel. To improve the sensitivity, a thermal isolation cavity is created underneath the flow sensor using the same sacrificial layer that creates the gap between the two electrodes of the valve. The heater uses the same metal layer as the top electrode of the valve. 


\section{FABRICATION}

The devices are fabricated using a 4-layer Parylene process with photoresist as the sacrificial layer and gold as the heater and electrode material. The process starts with a 4-inch silicon wafer. On the backside, Deep RIE etches through the wafer to make inlets and outlets for fluidic couplings. A $3000 \AA$ gold layer is evaporated on the front side as the ground electrode of the valve and wire bonding pads. A $1-\mu \mathrm{m}$ Parylene CVD deposition seals the electrode. This layer also serves as a buffer layer to improve adhesion between the successive Parylene layer and the substrate, because the presence of photoresist prevents the use of adhesion promoter later in the process. Then a $4-\mu \mathrm{m}$ photoresist sacrificial layer defines the gap between the two electrodes of the valve and thermal isolation cavity of the flow sensor. The top electrode and heater are formed by a $1500 \AA$ gold layer which is encapsulated between two $1-\mu$ m-thick Parylene layers. The fluidic channel is defined by a $5-\mu \mathrm{m}$ sacrificial layer and a 4- $\mu \mathrm{m}$ Parylene layer. Finally, the inlet and outlet holes are etched through the wafer, and the photoresist sacrificial layer is dissolved with Acetone.

Some process steps need special attention. Except for the first Parylene layer which uses adhesion promoter (A174 from Specialty Coating Systems), Oxygen plasma cleaning and roughening is performed before each Parylene deposition to improve the adhesion. The same is done when we deposit $\mathrm{Cr} / \mathrm{Au}$ on Parylene. Experiments show that prolonged Acetone soaking can result in delamination between Parylene layers. So as soon as most of photoresist is dissolved, the chips are transferred to Isopropyl Alcohol for further cleaning. The fabrication process is outlined in Figure 3. Figure 4 shows a micrograph of the fabricated device.

\section{TESTING AND RESULTS}

\section{Flow Sensor}

The flow measurement setup is similar to that of [6]. For airflow calibration, a constant pressure is applied to the inlet and a precision $10 \mu \mathrm{L}$ pipette is connected at the outlet. The volumetric flow rate is measured by the traveling speed of a bead of water in the pipette. For water flow calibration, a syringe pump is used to deliver a precise flow rate. The thermal sensor is operated in constant current mode. The ambient in the room is typically about $23^{\circ} \mathrm{C}$ and the fluctuation can be around \pm 1 ${ }^{\circ} \mathrm{C}$. To compensate for the effect of ambient or substrate temperature variations, outputs of two sensors with identical geometries are measured simultaneously. One of them is the actual flow sensor and the other is a compensation sensor located nearby. The difference between the outputs of these two sensors is taken as the final output for flow measurement. The temperature coefficient of resistance, $\alpha$, of the gold heater is measured to be $0.33 \% /{ }^{\circ} \mathrm{C}$.
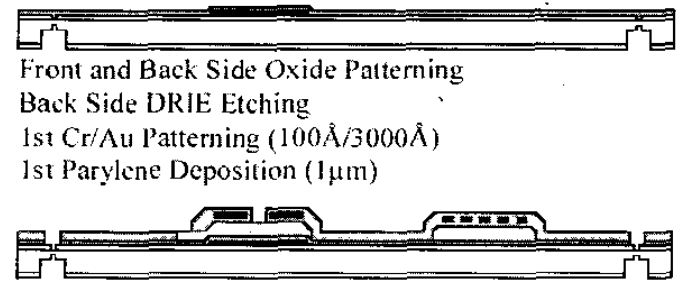

Ist Sacrificial Photoresist Patterning $(4 \mu \mathrm{m})$

2nd Parylene Deposition and Patterning $(1 \mu \mathrm{m})$

2nd Cr/Au Patterning ( $100 \AA / 1500 \AA)$

3rd Parylene Deposition and Patterning $(1 \mu \mathrm{m})$

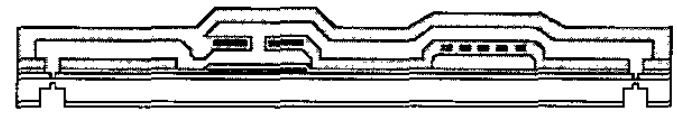

2nd Sacrificial Photoresist Patterning ( $5 \mu \mathrm{m})$

4th Parylene Deposition and Patterning (4 $4 \mathrm{~m})$

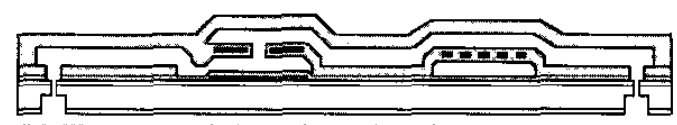

DRIE Open Backside Inlet and Outlet

Acetone Release Sacrificial Photoresist

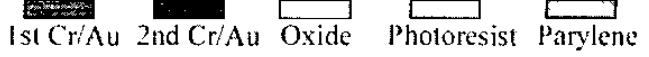

Figure 3. Fabrication process flow.

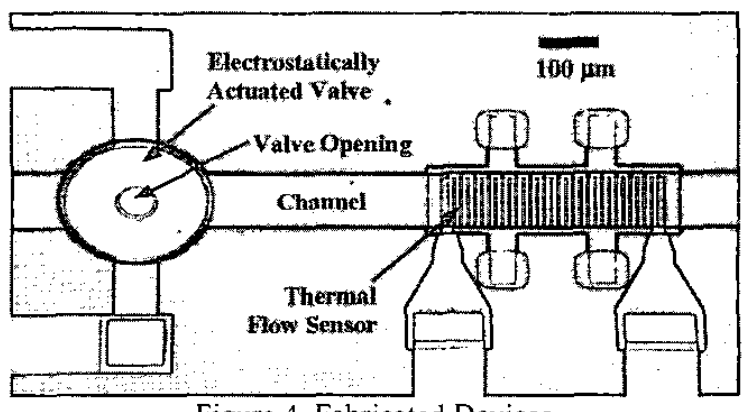

Figure 4. Fabricated Devices

Sensitivity $S_{\mathrm{v}}$ is defined as the ratio between output voltage change and flow rate. The relationship between temperature-to-flow ratio, TCR and sensitivity is:

$$
S_{V}=V_{0} \alpha \frac{\Delta T}{\Delta Q}=V_{0} \alpha S_{T}
$$

Figure 5 shows the voltage output as a function of airflow rate. A $4 \mathrm{~mA}$ bias current is applied, which corresponds to a heater temperature of $6.3^{\circ} \mathrm{C}$ above the ambient. Sensitivity $S_{\mathrm{v}}$ is $55 \mu \mathrm{V} /(\mu \mathrm{L} / \mathrm{min})$ and temperature-to-flow ratio is $0.033{ }^{\circ} \mathrm{C} /(\mu \mathrm{L} / \mathrm{min})$. Output voltage is measured with a HP34401A multimeter at a rate of $1 \mathrm{~Hz}$. The resolution of the voltage measurements is $10 \mu \mathrm{V}$. So the resolution of the flow rate measurements is roughly 0.2 $\mu \mathrm{L} / \mathrm{min}$. 
Figure 6 shows the flow measurement calibration for water. The bias current is again $4 \mathrm{~mA}$. The sensitivity is $12.2 \mu \mathrm{V} /(\mathrm{nL} / \mathrm{min})$ and the temperature-to-flow ratio is $0.0073{ }^{\circ} \mathrm{C} /(\mathrm{nL} / \mathrm{min})$. Water flow calibration proves to be more difficult. Due to the relatively large off-chip testing setup compared to the small flow rate, stabilization of flow takes a long time ( $>10 \mathrm{~min})$. This demonstrates the importance and advantage of on-chip flow measurement.

To study the dynamic response of the thermal flow sensor, the thermal time constant of the heater is measured. After switching on the $4 \mathrm{~mA}$ bias current, there is a nearly instantaneous jump to a voltage corresponding to the zero-bias resistance. The voltage, or resistance, change as a function of time, due to the heating, is given in Figure 7. The thermal time constant is measured to be about $1.0 \mathrm{~ms}$. Data is collected using a HP54645 digital oscilloscope.

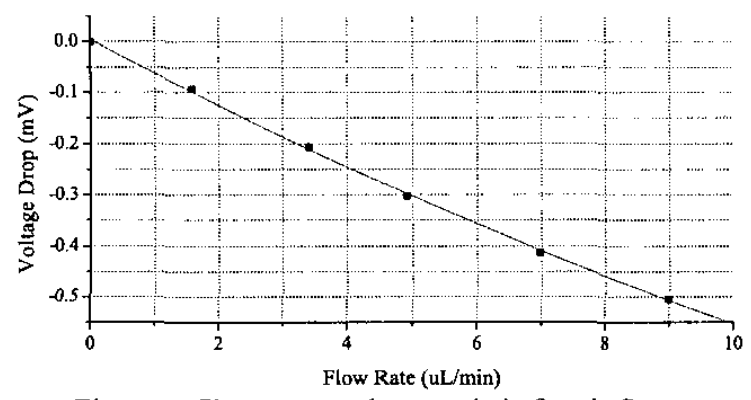

Figure 5. Flow sensor characteristic for air flow.

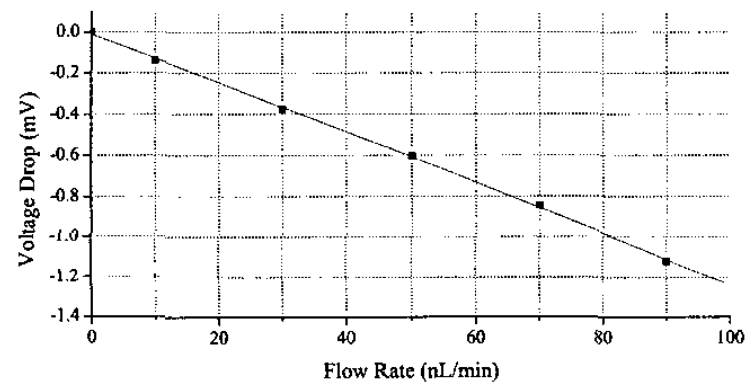

Figure 6. Flow sensor characteristic for water.

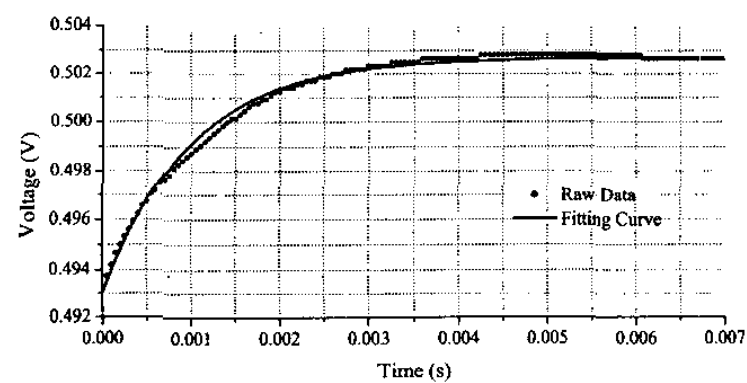

Figure 7. Flow sensor thermal time constant measurement

\section{Electrostatic Actuation}

To characterize the electrostatic valve, a voltage is applied between the two electrodes to actuate the valve membrane. The pull-in voltage is measured to be around
130 VDC. During the testing, we observe that after many actuations, the actuation strength attenuates. In addition, we observed a shift in pull-in voltage to as high as 180 VDC. Dielectric charging is the suspected cause of this shift $[4,5]$. To solve this problem, an $\mathrm{AC}$ actuation voltage is used, as suggested by [4]. A sinusoidal voltage is applied to the top electrode while the bottom electrode is grounded. A high frequency of $10 \mathrm{kHz}$ is chosen so there will be no oscillations of the valve at the actuation signal frequency. Using $\mathrm{AC}$ voltage, the pull-in voltage is about $110 \mathrm{~V}_{\text {peak }}( \pm 110 \mathrm{~V})$. The actuation strength remains stable in successive actuations. AC actuation is clearly better for our flow controller.

When the valve is actuated with water as the liquid, serious electrolysis problems are observed when using either $\mathrm{DC}$ or $10 \mathrm{kHz} \mathrm{AC}$ actuation. This occurs even though both electrodes are encapsulated with Parylene, which should provide electrical insulation. With water as the fluid, the valve membrane can be actuated using a DC voltage of around $20-40 \mathrm{~V}$. But almost immediately, bubbles are generated. Due to the rapid volume expansion of the bubbles, there is severe, permanent damage to the valve membrane. The valve cannot operate in water unless the electrolysis problem is solved. The flow control of liquids which have a high resistance to electrolysis, such as Fluorinert, has been demonstrated though.

\section{Micro Flow Controller}

In testing of the flow control capability of the system, two operation modes are investigated and compared. One mode is to adjust the actuation voltage amplitude. The other uses Pulse Width Modulation (PWM) [3]. In both cases, based on our calibrations, flow sensor output is used to measure the flow rate as we adjust the actuation parameters. Constant pressure is applied to the channel inlet to induce flow. Valve actuation signal frequency is kept at $10 \mathrm{kHz}$.

Figure 8 shows the relationship between output flow rate and actuation voltage amplitude. It is clear that as the pressure increases, the voltage needs to increase to stop the flow. When the pressure is higher than $28 \mathrm{kPa}$, the valve cannot be fully closed with voltages up to 220 $\mathrm{V}_{\text {peak }}$. Due to the positive feedback characteristic of electrostatic actuation, the linear region of the control curve is small.

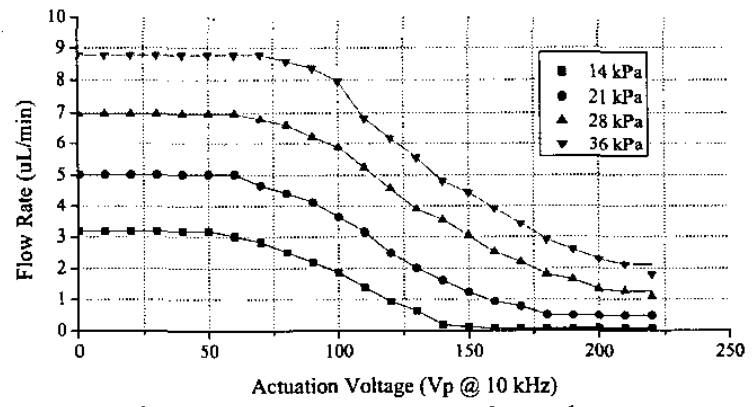

Figure 8 . Flow rate vs. actuation voltage. 
Since electrostatic actuation exhibits the pull-in phenomenon, it would be more beneficial to operate the valve in an on/off binary mode instead of the analog mode that is shown in Figure 8. To control flow rate, PWM is utilized. One requirement for PWM operation is that the valve has to switch fast enough so a relatively smooth flow is seen downstream. Figure 9 illustrates the control scheme. A pulsed modulation signal is applied to a solid state relay at $100 \mathrm{~Hz}$. The relay controls the valve by switching the $10 \mathrm{kHz}$ actuation voltage. The flow sensor output in Figure 10 shows system response to a $100 \mathrm{~Hz}$ control signal. Actuation voltage in Figure 10 is $160 \mathrm{~V}_{\text {peak }}$ and applied pressure is $34 \mathrm{kPa}$. System time constant is estimated to be around $1.5 \mathrm{~ms}$ and the valve actuation speed is likely on the order of $1 \mathrm{~ms}$ as well.

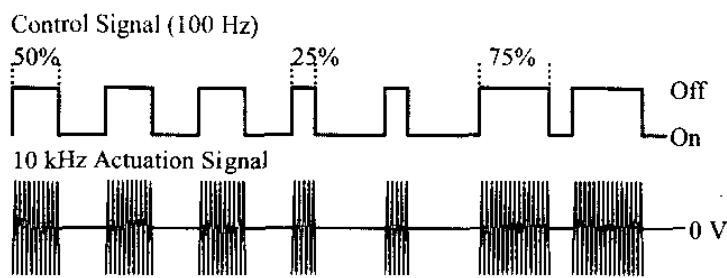

Figure 9. Control scheme in PWM mode.

Figure 11 shows the flow control results using PWM mode. The actuation signal is $200 \mathrm{~V}_{\text {peak }}$ at $10 \mathrm{kHz}$. The pulse control signal is $100 \mathrm{~Hz}$. For pressures lower than $21 \mathrm{kPa}$, the valve can be nearly completely closed. From $20 \%$ to $80 \%$ duty cycle, the control is quite linear. Non-linearity at both low $(<20 \%)$ and high $(>80 \%)$ duty cycles is caused by the fact that pulse durations are approaching the valve speed limit. Thus PWM operation becomes less ideal at both ends. Even with this nonlinearity, PWM control is still more linear than the control achieved by adjusting actuation voltage.

\section{CONCLUSION}

An integrated flow controller on a chip with an electrostatically actuated microvalve and a thermal flow sensor is developed. Together, they function as a micro mass flow controller. Fabrication of such a system is enabled by a multilayer Parylene process that we developed. Testing results demonstrate effectiveness of the design and flow control capability of the system for small airflow in the range of several $\mu \mathrm{l} / \mathrm{min}$.

\section{ACKNOWLEDGEMENT}

This work is supported in part by NSF ERC Center at Caltech (Grant No. EEC-9402726) and DARPA/MTO Bioflips Program. The authors would like to thank Mr. Trevor Roper for the assistance with fabrication and Dr. Yong Xu for the helpful discussion.

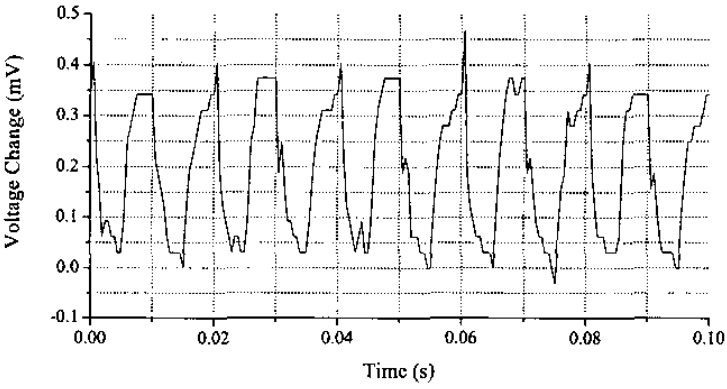

Figure 10. Flow sensor output when the valve is switched by $100 \mathrm{~Hz}$ control signal.

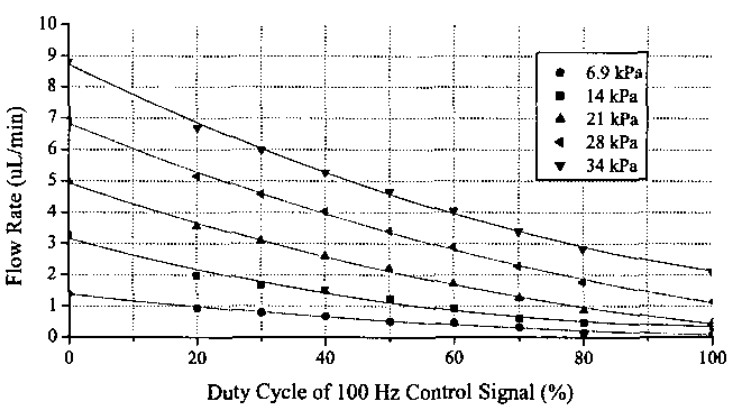

Figure 11. Flow control in PWM mode.

\section{REFERENCES}

[1] G.T.A Kovacs, Micromachined Transducers Sourcebook, McGraw-Hill, 1998

[2] J. Xie, X. Yang, X.Q. Wang and Y.C. Tai, "Surface Micromachined Leakage Proof Parylene Check Valve", The 14th IEEE International Conference on Micro Electro Mechanical Systems (MEMS'01), Interlaken, Switzerland, 2001, pp.539-542.

[3] P. Dubois, B. Guldimann, M.A. Gretillat, N.F. de Rooij, "Electrostatically Actuated Gas Microvalve Based on a Ta-Si-N Membrane", The $14^{\text {th }}$ IEEE International Conference on Micro Electro Mechanical Systems (MEMS'01), Interlaken, Switzerland, 2001, pp.535-538.

[4] W. van der Wijngaart, H. Ask, P. Enoksson, G. Stemme, "A High-Stroke, High-Pressure Electrostatic Actuator for Valve Applications", Sensors and Actuators A, 100 (2002), pp.264-271

[5] T.-J. Yao, K. Walsh and Y.-C. Tai, "Dielectric Charging Effects on Parylene Electrostatic Actuators", The $15^{\text {th }}$ IEEE International Conference on Micro Electro Mechanical Systems (MEMS'02), Las Vegas, USA, 2002, pp.614-617

[6] S. Wu, Q. Lin, Y. Yuen and Y.-C. Tai, "MEMS flow sensors for nano-fluidic applications," Sensors and Actuators A: Physical, 89 (1-2) (2001) pp. 152-158 